University of Tennessee Health Science Center

UTHSC Digital Commons

\title{
Maintaining the Master Patient Index: The impact of patient registration processes on data integrity
}

\author{
Matthew Biddle \\ University of Tennessee Health Science Center
}

Follow this and additional works at: https://dc.uthsc.edu/hiimappliedresearch

Part of the Health and Medical Administration Commons, Health Information Technology Commons, Health Services Administration Commons, and the Health Services Research Commons

\section{Recommended Citation}

Biddle, Matthew, "Maintaining the Master Patient Index: The impact of patient registration processes on data integrity" (2015). Applied Research Projects. 32. . https://doi.org/10.21007/chp.hiim.0016 https://dc.uthsc.edu/hiimappliedresearch/32

This Research Project is brought to you for free and open access by the Department of Health Informatics and Information Management at UTHSC Digital Commons. It has been accepted for inclusion in Applied Research Projects by an authorized administrator of UTHSC Digital Commons. For more information, please contact jwelch30@uthsc.edu. 
Maintaining the Master Patient Index:

The impact of patient registration processes on data integrity

Matthew Biddle

University of Tennessee Health Science Center

Health Informatics and Information Management

November 2015

Advisor: Dr. Sajeesh Kumar, PhD 
Abstract

The master patient index is one of the most important components within a healthcare system. It ensures that an individual patient is given a unique identifier that is used across the various separate clinical, financial and administrative systems and ensures that all information about that patient is organized and complete. Ensuring the MPI is accurate is critical since errors can have significant costs- both financially and in terms of patient outcomes. Patient registrars are the first line of defense when it comes to correctly identifying incoming patients and is where many errors in the MPI occur. Errors can be simple misspellings in a patient name, unknowingly assigning a patient a second medical record number, or the worst type, registering a patient under a different patient's MRN leading to intertwined medical histories being viewed by providers. This study looked specifically at a major health system in Richmond, Virginia to determine the existing workflows on how patients were identified in the MPI and how errors were corrected once known. A literature review was performed to determine if any evidence based practices exist for maintaining MPI data integrity and how the focus health system compares. In addition, two other comparable health organizations were surveyed to determine how they compare with the target and with national standards. It was found that the health system of focus did not have a MPI quality program in place at all which explains why it struggles with errors. A plan for a MPI program using criteria in the Improvement Focused Model was created and implemented. This plan focused on better collection of data on errors, more standardized procedures for registrars, and constructive feedback to registrars when errors do occur. Although after a relatively short period of implementation, there was little difference seen in the number of new errors being generated, it did find that there were a relatively few areas that were creating the majority of errors. Focus on these areas will undoubtedly help reduce future errors. Other factors that impact the lack of a decrease of errors is the complexity of how new registrations are created-some are generated by interfaces and have no human intervention at all. The takeaway from this study is that there are many more players with a critical role in data integrity than previously expected and that a quality MPI integrity program needs to be an ongoing program that focuses on continuous education, monitoring, and feedback to those players involved. 


\section{Table of Contents}

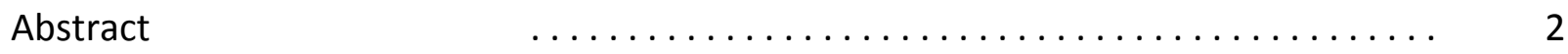

Chapter 1: Introduction $\quad \ldots \ldots \ldots \ldots \ldots \ldots \ldots \ldots \ldots \ldots \ldots \ldots \ldots \ldots \ldots \ldots$

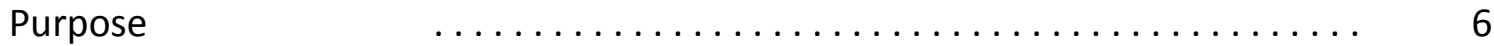

Background $\quad \ldots \ldots \ldots \ldots \ldots \ldots \ldots \ldots \ldots \ldots \ldots \ldots \ldots \ldots \ldots \ldots$

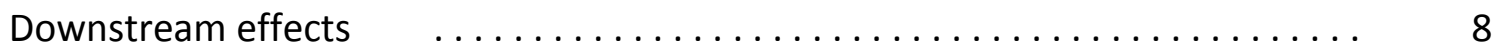

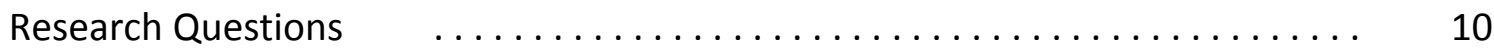

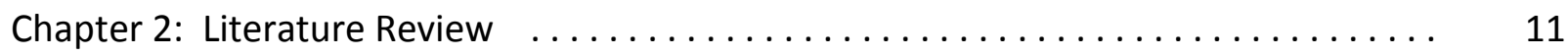

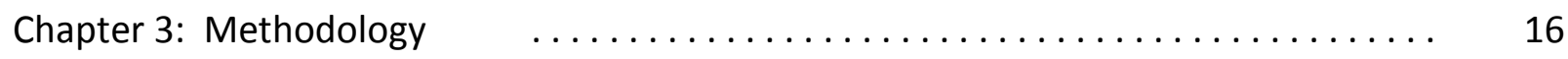

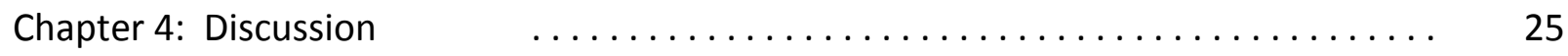

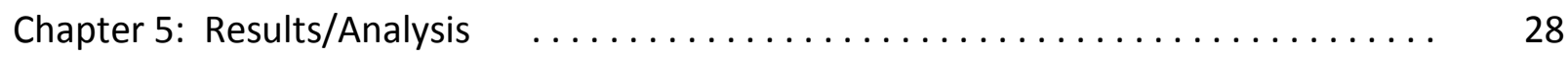

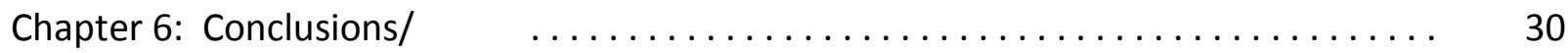

Recommendation

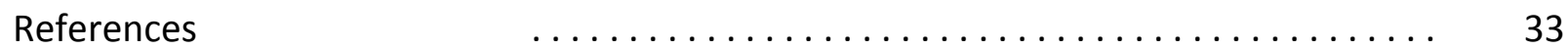

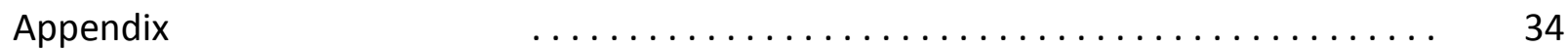


Maintaining the Master Patient Index: The impact of patient registration processes on data integrity

\section{Chapter 1}

\section{Introduction}

The master patient index can be considered one of the most important components within a healthcare system. The master patient index or MPI identifies a single patient with a unique identifier across the various separate clinical, financial and administrative systems within a healthcare organization. The MPI allows for a patient to be represented only once so that a complete picture can easily be seen. Simply put, the MPI is a very large table or database that includes patient information such as patient name, date of birth, gender, social security number, address, phone number, insurance information and other data used in the registration and billing processes. Each individual patient has a unique identifier, usually referred to as the medical record number. Each time the patient returns to the same healthcare facility, the patient will always have the same medical record number (MRN). This allows all information about that patient to be kept in an organized and easily usable fashion which ultimately results in efficient treatment and payments.

Maintaining a clean and accurate MPI is a task of extreme importance since patient's medical histories are usually linked to their MRN. An inconsistency in identifying the patient correctly in the MPI can lead disruptions in the treatment of that patient causing inconveniences and even harm. Unfortunately, errors in the MPI are something most 
healthcare facilities deal with often. In fact, Lynn Kuehn (1997) showed that there is plenty of evidence that shows that most healthcare facilities are seeing error rates in their MPI of between five and ten percent. Errors are usually seen in the form of a single patient being added multiple times resulting in multiple MRNs. This type of error is known as an overlap. The other type of error, known as an overlay is a bit more serious and is where two different patients share a single MRN, intermingling their medical information.

Typically, it is the job of the Health Information Management department to maintain the MPI. When an error is found, it is their job to do an in-depth analysis to determine the best plan of correction. If the error is found to be an overlap, the multiple MRNs for the same patient will be merged together so that only a single active MRN exists. If the error is an overlay, a deeper investigation must occur to ensure the two unique patients and their medical information are separated correctly and each assigned to their own MRN.

There are many studies on the topic of MPI maintenance and essentially they all indicate there is a critical need for a quality program to search out potential errors so they can be corrected-not just a passive program that corrects errors as they are found when patients get registered but a program to actively search for and eliminate existing errors in the MPI. Errors in the MPI tend to "snowball" creating more problems along the way that must be corrected which is why this aggressive approach is needed. Fortunately, as Altendorf (2007) indicates in her article," Establishment of a Quality Program for the Master Patient Index", that as technology has evolved, so too has the MPI which is no longer maintained on paper catalogs and exists as an electronic database in healthcare facilities today. The advantage of this is the 
ability to query the MPI database to look for certain types of errors- potentially a game changer in the MPI world.

Managing the integrity of patient information is nothing new. However, new opportunities and options have exploded in the healthcare field over the decade. Legislation such as the American Recovery and Reinvestment Act of 2009 has been a major contributor for the better use of technology in healthcare. With this push came new sets of requirements, standards and thresholds that had to be met and surpassed with the goal to help improve patient outcomes, increase efficiency, and lower costs. Behind the scenes, these forward movements have meant massive amounts of data are now being collected and analyzed that were never available before. The master patient index is a perfect example and as such is ripe with data for the taking.

\section{Purpose-}

The purpose of this study was focused on improving the entire process of MPI maintenance and error prevention at a specific healthcare facility located in Richmond, Virginia. Virginia Commonwealth University Health System (VCUHS) is located in the heart of downtown Richmond, the state's capital. A medical school is a major component of this teaching hospital. The facility boasts a 600 physician faculty group practice, 1,125 inpatient beds and over 200 outpatient specialties. It contains the area's only designated cancer center and is the region's only Level 1 trauma center making it a referral center from facilities all over the state and 
IMPACT OF THE PATIENT REGISTRATION PROCESS ON DATA INTEGRITY

beyond. In 2014, the health system saw over 35 thousand inpatient discharges, 84 thousand emergency department visits, 615 thousand outpatient clinic visits and performed over 21 thousand surgeries (VCU, 2015). The high patient volume indicates that the health system has many opportunities for errors in the MPI.

\section{Background of the problem-}

The master patient index at VCUHS, like other established facilities, began on paper and has since transitioned over to be maintained in an electronic database. The health system's sheer size has made implementation of any type of enterprise wide changes very difficult and slow. As a result of this, VCUHS lags behind other healthcare facilities with respect to electronic health record and interface technology. One of the major sources of problems can be attributed to the fact that the health system uses a software package called GE-IDX to register patients and a second software package produced by Cerner to act as the electronic medical record. Interface transactions between those software packages and the MPI are supposed to keep everything in sync. The reasoning behind the decision for separate systems has to do with very specific differences in the capability of each software package. I.e. GE-IDX functions better in registration but not as well as an EMR and vice versa for Cerner. However, because of the varying capabilities of the software packages, each has different procedures in how to make corrections. When an error such as an overlap or overlay is found, existing processes to make the correction are convoluted, tedious, and slow. In addition, the corrections must be made in each system individually because of the differences in how they 
accept changes. Because of the tedious nature of the process, the Health Information Management staff tasked with making MPI corrections is overwhelmed and only passively makes corrections when they are found by a registration clerk when a patient arrives. This has led to a backlog of known errors waiting to be corrected which, as stated above, is a recipe for inefficient and ineffective treatment and billing outcomes.

\section{Downstream Effects-}

A clean and accurate MPI is a critical necessity to be able to take full advantage of newly available tools such as Health Information Exchanges (HIEs) - whereas information is shared, usually with a third party, which then acts as a hub of information which can be requested. Information within HIEs and EMRs must be accurate in order for it to be complete and useful. Beth Just of AHIMA (2009) indicates that seemingly mundane errors such as transpositions in a patient's date of birth, name misspellings, use or nicknames or default social security numbers can wreak havoc in the successful linking of patient records within and across various health information systems and health care facilities. Often, information is sent between providers using a unique patient identifier such as a MRN as the basis of an electronic transaction and uses other additional demographics to validate. When that information doesn't match between providers, often the information is treated as a new and unique patient even when the patient is already present in the MPI resulting in duplicate listings of the patient, each with different information available in the medical record leaving a huge hole that can affect patient treatment. So far, health care legislation for the integrity of patient identity is non-existent. 
Existing statutes focus on standardization of the data format; however, the accuracy of the data content has not been addressed.

Point blank- errors in the MPI lead to poorer patient outcomes and come with substantial costs. Specifically, duplicates in the MPI-having a single patient with more than one MRN- can lead to medical decisions made based on incomplete information, duplicate procedures performed, reimbursement denials, and potentially medical malpractice risks. To make matters worse, an AHIMA study performed by Mike Basset (2011) found that nationwide, hospitals have a duplicate patient record rate of ten percent. Other studies (Just Assoc., 2011) have found that each duplicate patient record costs the organization \$96 just in administrative costs to fix the errors. Additionally, repeat tests or treatment delays cost an average of $\$ 1,100$ each and $4 \%$ of confirmed instances of duplicate patient records had a direct impact in critical care to the patient.

Specifically, at VCUHS, each error in the MPI causes additional headache in the process of fixing it because of the unique network of various patient software systems that exist. We have separate software packages for the EMR, registration, radiology, cardiology, operating room, pathology and others. The process to fix just one duplicate involves multiple steps and cooperation among several departments other than HIM making the focus on preventing these errors on the front end, the main goal. 


\section{Research questions-}

This research is intended to be an observational study in the current processes and policies surrounding the master patient index both at VCUHS and other comparable health care facilities in the area. The purpose is to understand how each facility maintains their prospective master patient indices including a look at what processes are in place to prevent errors in the MPI and the processes to locate and correct errors once they exist. This topic was chosen specifically to understand why VCUHS struggles with errors in the MPI, to determine if other comparable facilities have made any improvements in their processes and to discover if any changes could be made to bring the processes more in line with industry standards.

The premise of this research is simple- to determine what the national gold standards are and to determine if local healthcare facilities are utilizing them to the best of their ability and if not, to determine how changes can be implemented to bring them more in line with the standards. 


\section{Chapter 2}

\section{Literature Review}

Data integrity is of utmost importance. Robin Altendorf in his 2007 publication "Establishment of a Quality Program for the Master Patient Index" indicates a typical successful program aimed at maintaining this data integrity uses an Improvement Focused Model as seen in Figure 1 below. This model indicates the major components of a successful MPI program include assessment, education, review, monitoring, maintenance and mentoring.

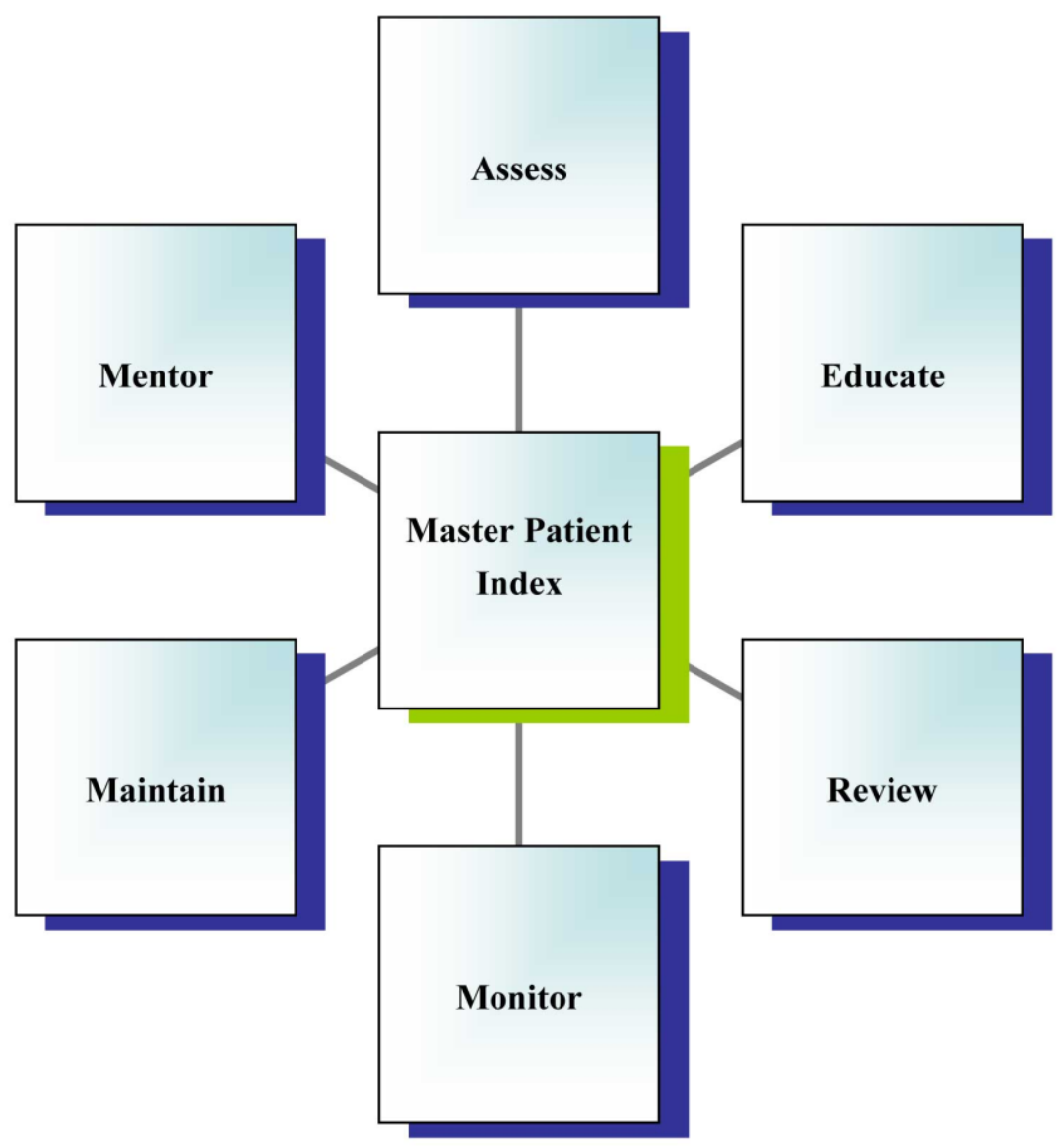

Figure 1. The six components of an Improvement Focused Model of a Quality MPI program. 
Generally, an assessment of the MPI process is done to provide a baseline on where an organization stands with MPI and registration errors and processes. The assessment phase is to determine the current levels of errors in the $\mathrm{MPI}$, the registration process and how errors are occurring. Understanding the patient registration process is critical. Correctly identifying each and every patient the first time is the ultimate goal for each registrar- but the reality is that simply does not happen $100 \%$ of the time. This can be due to human error or to processes in place that leave room for variances in capturing all necessary information.

In 2007, the World Health Organization along with the Joint Commission indicated that patient misidentification is one the most prevalent root causes for treatment errors and patient harm. It laid out a list of suggested actions to help eliminate these errors which points to the next component in the Improvement Focused Model- education. These actions were: 1) Emphasize to patient registrars that their primary responsibility to correctly identify patients. 2) Require the use of at least 2 identifiers to verify identity. 3) Standardize the approaches to patient identification across all points of registration in a health care system and 4) Encourage patients and families to actively participate in the identification and data integrity processes.

Beth Just (2009) takes this a step further and explains that not only are actions like these necessary as a one-time orientation of new employees but rather these items must be a part of a much bigger program of ongoing and focused management and education. These ongoing education programs must emphasize in great detail exactly how patients should be identified when they show up to a registration desk. Generally, two patient identifiers (usually the patient's name and date of birth) are seen as the absolute minimum that should be 
obtained by the registrar in order to search for a patient in the master patient index. However, it should be just that- a minimum- and should be a goal to ask for additional information to confirm the correct patient has been located in the MPI. When a patient cannot be located in the MPI using the patient provided information, generally a new medical record number is generated. Prior to creating a new MRN, the registrar should be sure they are not creating a duplicate for that patient. Often, simply asking the presenting person if they have ever been a patient at the facility is enough to prompt more information that can be used to confirm the person is or is not a patient that would have an existing MRN. Playing devil's advocate, Just (2009) also recognizes that the registrar should understand that the emphasis on limiting creating duplicate MRNs does not influence the registrar to the greater evil of registering a patient under a different patient's thereby blending the medical information of two different patients together.

A quality MPI program using the Improvement Focused Model will also have ongoing reviews of existing policies and procedures that focus on determining if errors are still occurring after staff education. This is an opportunity to revise an education of staff originating errors or if there are other areas such as revising data fields or search methods in the MPI that can be tweaked. Altendorf (2007) also indicates at this point, that the program coordinator should know if the registrars have the skill set and understanding to continue to function in the tasks. The next component of the quality MPI program should have ongoing monitoring of new MPI errors and staff performance. A routine report of corrections should be communicated to those departments responsible so affected downstream systems that may contain the same errors can be corrected. 
The next component of a successful MPI program is routine maintenance, often by auditing the MPI for errors. Usually in the form of a software package or a query or even a vendor service, an audit of the MPI uses pre-established parameters to search the MPI to help identify existing errors. Beth Just (2009) found that most audits of the MPI use at minimum a basic algorithm technique for matching records. In this instance, parameters such as the name, birth date, SSN and sometimes gender are used to scan the MPI. Within this basic algorithm, an audit can be run to find exact matches or even matches that sound alike or are close in spelling but not exact. An example of an exact match would be a query looking for duplicate patient records that have the exact same SSN while a determinate match would return results for patients with last names of "Johanson" and "Johnson" as potential duplicates since they may sound similar. Lastly, a wild card match is usually one that returns potential duplicates in which the first few letters are the same but the last letters may be different. For example, a wildcard search on last name "John*" would give results including patients with last name "John" and "Johnson". Any of these queries could be used in conjunction with each other or separately. Altendorf also indicates more advanced algorithms that are sometimes used in instances where a search can assign a higher weight to certain fields such as SSN and a lower weight to fields such as gender. The most advanced algorithms used in MPI audits use sophisticated mathematical theories to indicate a probability that a match is a true duplicate. Regardless of the type of MPI audit performed, the results should be verified by staff, usually in the Health Information Management department, to determine if a match is a true duplicate/error before attempting to merge or correct it. False negatives and false positives 
will always occur which is where substantial training and ongoing education help staff make the correct determination.

The last component and arguably the most important is that of mentoring. This is often times in the form of feedback to those individuals responsible for errors in the MPI. This is an opportunity for management to provide constructive criticism to help staff be proactive in their jobs. As a side effect, open dialogue in a constructive manner, ensures everyone is interpreting procedures the same way and helps decrease high turnover in registration staff that also contributes to errors in the MPI.

Overall, MPI integrity is a critical piece of the patient treatment and revenue cycle processes. The risks of lost revenue or even patient harm are major drivers for health care facilities to implement programs such as these. Just (2009) concludes that quality patient data programs cleanly aligns with what management experts have been voicing forever- that you can't manage what you can't measure. This is essential to overall healthcare reform, improving the quality of care, increasing administrative efficiency and reducing costs. Six sigma accuracy (99.999999\%) might not be the reality today for MPIs in healthcare organizations but striving for that level of quality is not just for the airline industry anymore. 


\section{Chapter 3}

\section{Methodology}

\section{Patient Registration at VCUHS-}

Virginia Commonwealth University Health System is a large teaching facility in Richmond, Virginia and as such is a major hub for most of central Virginia being the region's only Level 1 trauma center. The health system not only includes the main hospital facilities but also over 200 outpatient specialties among its 600 physician group practices with about half of those being in stand-alone outpatient clinics all over the state. Because of this, it was identified that initial patient contact was made in the following ways: 1 ) through the emergency room either by walk in or by ambulance, 2 ) the communications center responsible for scheduling patients who call in by phone, 3) patients who show up at or call directly and of the 200 outpatient clinics, and 4) transfer from another hospital.

Although each arrival method has slight variations, generally, when a patient initiates contact with VCUHS, the procedure is as follows. Upon contact, the registrar asks the patient for their name and date of birth. The registrar begins a patient search of the MPI in the registration software called GE-IDX. An example of the patient search screen can be seen in figure 2. The registrar typically uses the full last and first name along with the DOB to search. If a patient is found in the search results, the registrar will ask the patient to confirm additional information such as their SSN and current address and will compare them to the patient found in the search results. If the name and date of birth search do not return any results, the registrar will generate a new MRN. After the patient is either located in the existing MPI search 
results or a new MRN is generated for them, the registrar will obtain the rest of the current demographic and insurance policy information needed for a full patient registration profile.

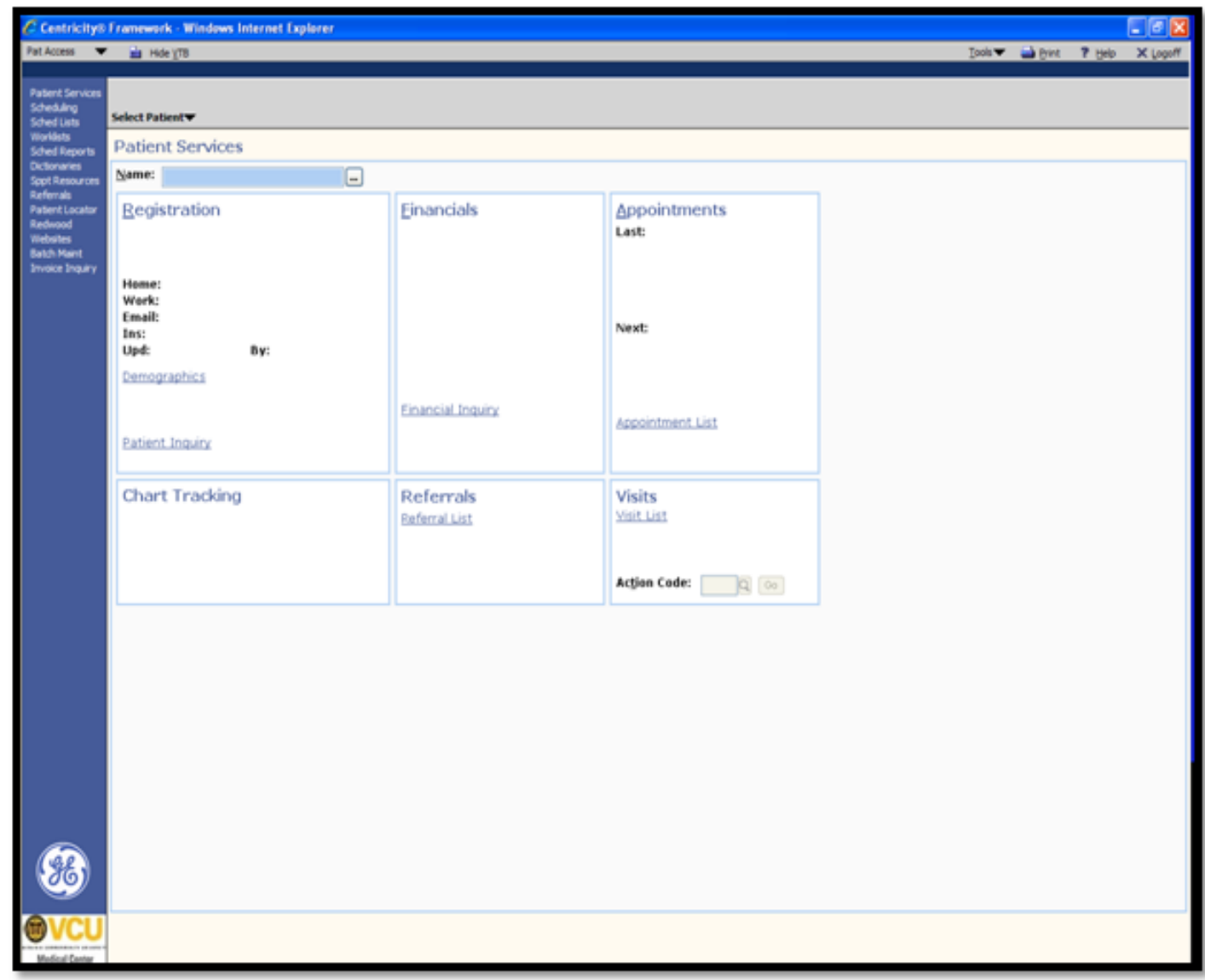

Figure 2. Patient search screen in GE-IDX registration software used by VCUHS

Variations to the standard registration process occur in several instances depending on how they make contact with the health system. The ER is unique in this aspect. The major difference is that an entirely different software system called Cerner (our EMR software) is used to register patients that arrive in the ER instead of the registration software, GE-IDX. The decision to use the EMR to register patients is due to the emergent nature of many patient that arrive in the ER and the ability to create pre-emptive orders based on the patient's chief 
complaint. Another unique piece of the ER registration process is the difference when the patient arrives via ambulance versus walking in. Arriving via ambulance, the patient information is called in prior to arrival by the EMS agency and a "pre-reg" is completed by ER staff which is simply a temporary placeholder with a name, date of birth and a temporary MRN. Only after the patient has been seen by a physician and considered in stable condition will a registrar go visit the patient in a bed and do a full registration.

One other variation is that which occurs when a patient calls in to schedule an appointment. During a phone call, a driver's license/identification card cannot be obtained to show the spelling of a patient name and therefore the communication staff must rely on patient pronunciation and phonetics to collect the information and complete the patient search of the MPI. Figure 3 shows the registration process and how it differs depending on how the patient makes contact with VCUHS. 


\section{Patient Registration Process}

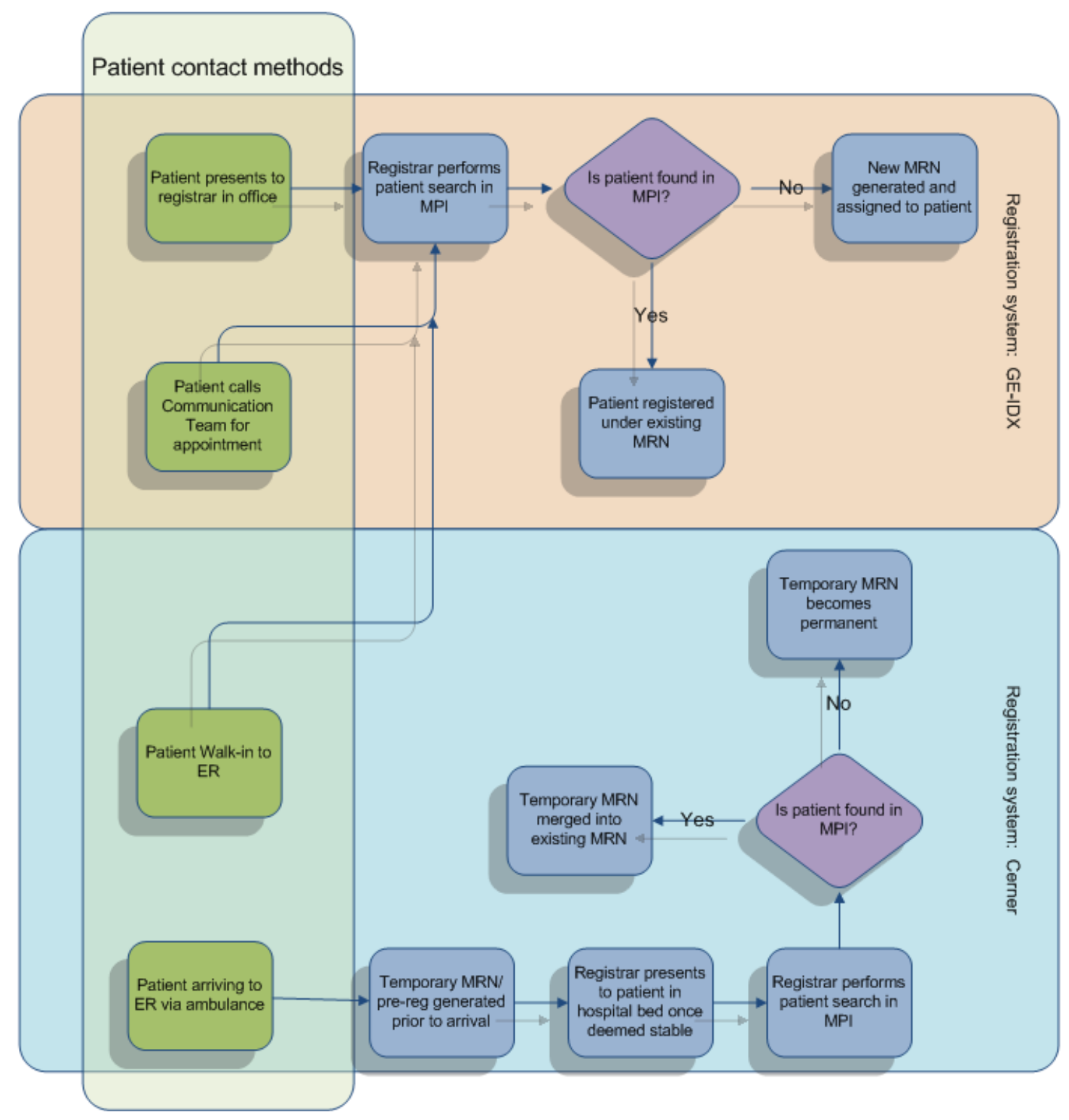

Figure 3. Shows variations in the patient registration process depending on how the patient makes contact

The other half of the MPI process is the identification and correction of errors. This is the part that VCUHS struggles with. The identification of errors occurs in several ways. The majority of errors are found on what we refer to as the back end- where a duplicate or 
misspelling is found when a patient is being registered during the patient search of the MPI. When a duplicate is found, the registrar/scheduler will notify the HIM department of the duplicate and staff on the data integrity team will investigate and confirm the error and make the correction which is usually a merging of the two MRNs into one and updating all the demographics to the most current available. This merge can only be completed if the registrar has not "arrived" the patient. "Arrived" is the flag that indicates that the patient is in house and currently being seen by a provider indicating an encounter is open. A merge while a patient has an open encounter will disrupt treatment since the patient identifying armband may now have a different MRN than any provider documentation, blood tests or radiology reports. Since a majority of errors are found by registrars, the data integrity team is inundated by telephone calls and emails on a daily basis. The other avenue of identification of errors is through a subpar reporting system. There is an MPI audit report that exists to notify the data integrity team of potential errors but the reason this is subpar is threefold-1) the parameters of the query that search the MPI are too loose producing many false positives that must be investigated, 2) the query results are triggered when anyone "touches" the registration screen regardless of whether a MRN was just created or was created 20 years ago and 3) there is no way to remove or flag a potential duplicate result that is known to actually not be a duplicate. All three of these together mean that the daily report contains a lot of "garbage" of false positives or known patients that are confirmed not to be duplicates that repeatedly show up with no way to flag them.

Lastly, the actual correction process by the data integrity team is not a simple procedure. An error such as duplicate MRN, once known, is investigated, confirmed and finally 
merged together so that the patient only has a single, active MRN. The merge process begins with launching a merge application in the EMR system, locating both MRNs for the patient and indicating which should be the primary number and finally the secondary MRN is merged into the primary. It gets more complicated since both Cerner (as our EMR) and GE-IDX (as our registration system) are used, the process has to be completed in both-- however, GE-IDX doesn't have a merge function, only a deactivation function. So the secondary MRN is deactivated in GE-IDX and the primary MRN is notated. Lastly, a third software system that is our image repository, must be examined to determine if any scanned documents need to be moved from the secondary MRN to the primary MRN. Figure 4 shows the current workflow for the data integrity team at VCUHS for locating and correcting a duplicate medical record number error. 


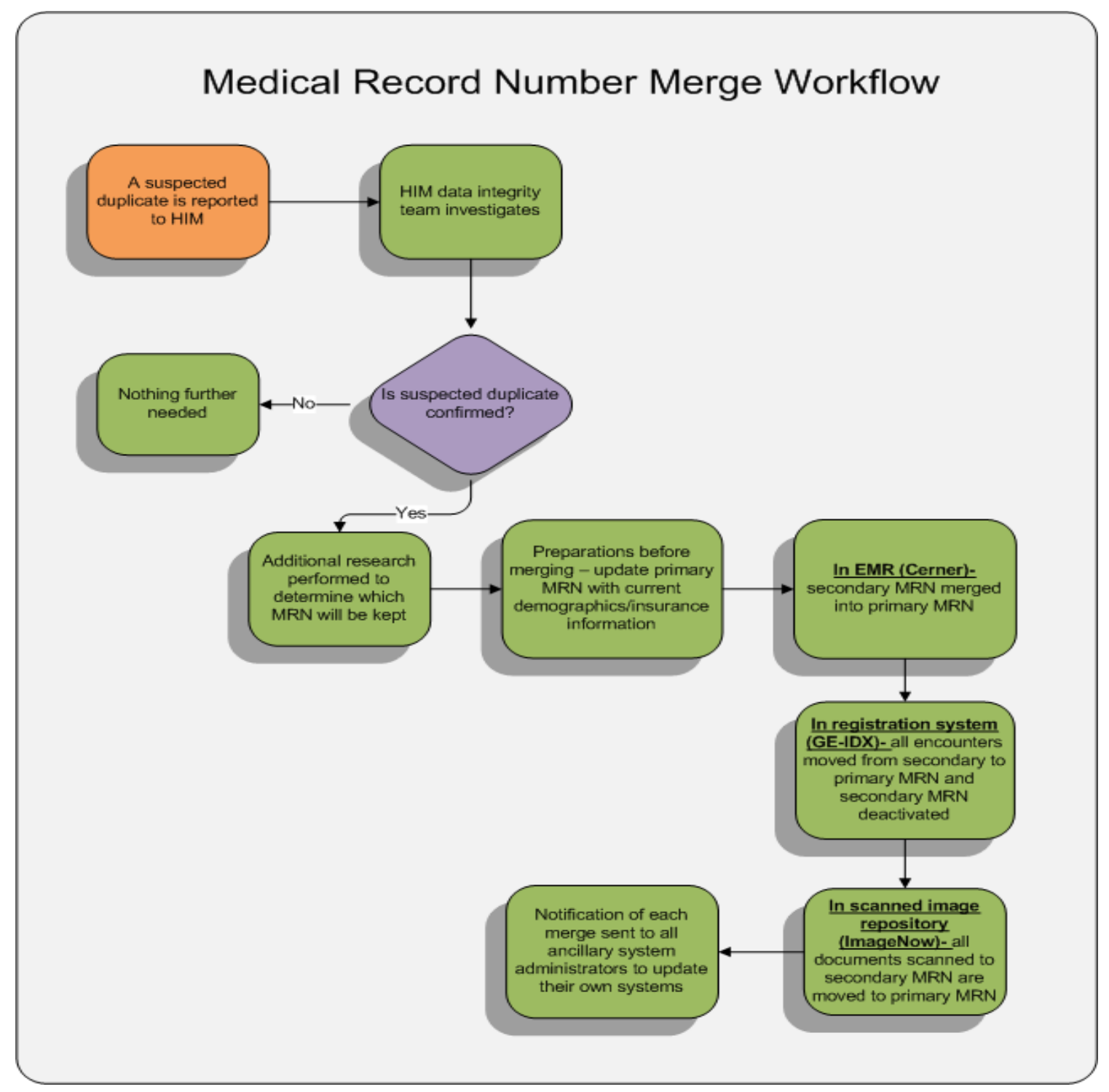

Figure 4. Shows the workflow used by VCUHS to locate and correct an error in the MPI

At that point, the merge process for HIM is complete but then every other software system in which these merge transactions don't directly interface must be updated. An email group containing some 40 people responsible for all the various systems (examples of specialty systems include: radiology imagery, pathology, operating room, anesthesia) are notified to ensure the MRNs are updated. Generally, this is seen as a clunky and tedious process and 
therefore the focus has been placed on the prevention of errors rather than the cleanup. Appendix A offers a look at the vast network of systems and file transfers that exist at VCUHS that must work together to make sure data is consistent and accurate.

\section{Comparables-}

There were two other healthcare organizations that were polled regarding their registration processes in relation to data integrity. Both of these were located in the central Virginia area and each was comparable to VCUHS in different ways. The first comparable was the University of Virginia Health System located in Charlottesville, Virginia about 75 miles west of VCUHS. Like VCUHS, UVAHS is a teaching hospital. It is slightly smaller in size at 584 beds but is still a major system for the region considering it is located in a more rural area. The second comparable was chosen because of its proximity to VCUHS- Johnston-Willis Hospital located in Richmond, VA, just 12 miles away. This hospital is much smaller at 292 beds but is a part of a 6 hospital system in Virginia belonging to the well-known HCA hospital network.

Surprisingly, Johnston Willis Hospital was the only facility boasting a $100 \%$ paperless electronic medical record. Their EMR software was MediTech and it was a single software package used for medical records and for registration. On the other hand, the EMR of UVAHS, was the well-known Epic software which was used for both registration and for EMR capabilities, although they are currently in a paper-electronic hybrid state. The handling of medical record errors also had minor differences between the two. UVAHS relied on a system query of their MPI using an algorithm demonstrated by Beth Just (2009) to search for likely 
duplicates which were placed on a queue daily for HIM staff to further investigate and correct if necessary. JW differed slightly in that an MPI query was completed ad-hoc and more often relied on registrars to notify HIM of duplicates and errors when found while registering a patient. This is very similar to VCUHS. Because of their ability to query the MPI using whatever parameters desired, both of these facilities were able to run reports as needed to see trends over time of errors. These allow both facilities to give feedback to registrars- which they both do- who may be responsible for creating the errors unnecessarily, something VCUHS is not very good at. These results can be seen in table 1 below.

Table 1. Comparison among three facilities in their software and registration processes

\begin{tabular}{|l|l|l|l|l|}
\hline & VCUHS & UVAHS & JWH & $\begin{array}{l}\text { Goal (if } \\
\text { applicable) }\end{array}$ \\
\hline EMR software package & Cerner & Epic & Meditech & N/A \\
\hline Used for registration? & No ; IDX & Yes & Yes & N/A \\
\hline EMR stage? & Hybrid & Hybrid & $100 \%$ electronic & $100 \%$ electronic \\
\hline How are errors found? & Passive & Active & Passive/Active & Active \\
\hline Are errors tracked? & No & Yes & ad-hoc reporting & Yes \\
\hline Registrar training & orientation & as needed & as needed & $\begin{array}{l}\text { on-going } \\
\text { scheduled updates }\end{array}$ \\
\hline Is feedback given? & No & as needed & as needed & $\begin{array}{l}\text { on-going } \\
\text { scheduled updates }\end{array}$ \\
\hline
\end{tabular}




\section{Chapter 4}

\section{Discussion}

After analysis of the known pitfalls of the current processes and comparing them to the national standards as well as the processes of other local facilities, a plan was created to implement changes to better mimic those industry standards. Following the Improvement Focus model, the key areas identified to be areas for improvement were: 1 ) the patient registration process, 2) registrar education, 3) reporting.

To improve the patient registration process, registrars needed to add an extra step to their patient search process. When they search by the two patient identifiers, name and date of birth, and no results are found, prior to them generating a new number, the process should include simply asking the person if they have ever been a patient at the facility before and a third identifier that can be used for a second search. For example, if the name and DOB return no matches but the person indicates they have been a previous patient, then a SSN should be obtained and a simple search only on that field should be completed. Often times a simple misspelling of the name will result in a non-match while the SSN will find the patient in the MPI. The deliverable for this part was working with the revenue cycle training team to improve training manuals and creating an online module for registrars that will relay updates and improved workflows as they are found. Once completed, managers of registrars were given training materials to educate on standardized methods of searching for patients and the proper procedures that should be followed before a new MRN is generated. 
The education component is related to both the registration process improvement and the reporting component. Registrars should undergo more than just an initial on the job training session when they are hired and instead should undergo ongoing re-education sessions where they can be shown new procedures and create an open dialogue with their managers who should be providing feedback from what is found in the reporting component. The deliverable for this piece was that the online training modules created by the revenue cycle training team will be a part of every registrar's annual competency education. These modules will be updated as needed as new options and capabilities are made available with upgrades to the EMR and registration systems.

Lastly, improvements in the reporting capabilities and processes need to be established. This is twofold: 1) the existing MPI audit report needs desperate improvements in the parameters that it uses to query potential duplicates, in the ability for HIM staff to flag false positives. This will require an IT resource and will likely be considered low priority. And 2) there is currently no feedback at all given to the registrars who create MPI errors. Countless studies such as Chris Dimick (2009) in his AHIMA publication "Exposing Double Identity at Patient Registration" indicates that providing feedback is critical. Registrars who don't know they are making errors have no reason to stop. The deliverable for this component is that the HIM will work with IT to develop a better MPI audit report. There is no short term solution to make this happen so in the meantime, a manual process of creating a shadow report of true duplicates will be a new task of the data integrity team. This temporary workaround will allow the known duplicate backlog to be monitored and will provide accurate data that can be 
provided back to the registrars as feedback in an attempt to prevent the same mistakes from continuously repeating themselves by the same registrars. 


\section{Chapter 5}

\section{Analysis of findings/Results}

The improvement focused plan is just in its beginning stages but after initiating the implementation of most of the recommendations, there were some observable changes although not all was expected. Ultimately, there was little change in the number of new incidents of duplicate MRN creation as seen in Table 2.

Table 2. Shows total duplicate MRNs created in the MPI for the first 3 quarters of 2015

\begin{tabular}{|l|r|r|}
\hline & Total duplicates & Average per day \\
\hline Q1 2015 & 543 & 6.0 \\
\hline Q2 2015 & 666 & 7.3 \\
\hline Q3 2015 & 686 & 7.5 \\
\hline
\end{tabular}

When adjusting for any potential changes in the number of patients the results are still less than stellar as seen in Table 3. In this case, discharges included are all patient types except OP physician clinic visits. Note that the number of discharges climbs throughout the year but so does the percentage of duplicates. 
Table 3. Shows total number of duplicate MRNs created compared to number of patient discharges

\begin{tabular}{|l|r|r|r|}
\hline & Total duplicates & Total discharges & Duplicates/discharges \\
\hline Q1 2015 & 543 & 49,573 & $1.10 \%$ \\
\hline Q2 2015 & 666 & 51,975 & $1.28 \%$ \\
\hline Q3 2015 & 686 & 52,369 & $1.31 \%$ \\
\hline
\end{tabular}

Analysis of the new errors created daily found that it was a relatively low number of clinical locations creating the majority of the errors. In fact, just three locations were responsible for $51 \%$ of all errors in the third quarter. The top 10 offending clinical locations were responsible for $83 \%$ of all duplicate errors. When considering the healthcare system has over 200 locations, this is good news that just a handful of areas can be focused on for future follow up. 


\section{Chapter 6}

\section{Conclusion/Recommendations}

Ultimately, this project was intended to fully understand all aspects of the best practices surrounding the master patient index and to see if they were actually being implemented at several local organizations. The results in the tables above showing little improvements should be seen as only a part of the picture. To put it bluntly, the software interoperability at VCUHS and the processes in place to work around it is simply a lot more complex than other facilities and is has a major impact on efficiency. There are multiple registration systems, multiple levels of training of different staff using the systems, and multiple other systems that are not fully interoperable with each other- all of which are creating varying opportunities for errors.

There does not seem to be any national statute listing a specific target error rate, however, the healthcare industry generally holds that an error rate of less than eight percent is normal (Morris \& Farnum, 2014). This error rate applies to all types of errors in the MPI including misspelled names or incorrect DOB, while this project was mostly focused on duplicate MRN errors. To make matters more complicated, Basset's (2013) article indicates that there isn't a clear national standard on how to measure duplicates. For this project, duplicates were queried daily based on parameters of similar first names and exact matches on last name and date of birth. The numbers of duplicates were compared to the number of patient discharges during the same time period. The number of admission/registrations would 
have been a better indicator but this data was not easily obtainable. The duplicate rates of between $1.1 \%$ and $1.3 \%$ should be considered the rates of new duplicate incidents and not the prevalence of duplicates that exist in the entire MPI, but it is a good sign that current workflows are keeping new duplicate rates much lower than the industry accepted $7 \%$.

For this project, it was very clear from the beginning that evidence based practices should always be consulted and used when an organization is developing its own plan. In the case of VCUHS, it was found that there were huge gaps missing from the workflows to help eliminate duplicate medical record in the MPI. Comparing our own processes with those described in the Improvement Focused Model supported by Altendorf (2007) and with other publications in AHIMA's body of knowledge of a quality program of MPI data integrity, it was clear own procedures needed to be adjusted. Specifically, holes were found in the patient registration process, registrar training/education, and reporting/feedback. This is very significant because these are essentially the three cornerstones of what a data integrity program should have-- and astoundingly, these components were absent.

The takeaway from this project was that there are many more players that have a critical role besides HIM in data integrity than originally expected. Starting with the registration staff- they have a much more active role in the process than simply typing in the patient's name correctly. They need to be fully educated on all the patient search options, know how and when to ask probing questions, and have critical thinking skills to properly analyze situations that seem out of the ordinary. On top of this, because there are ongoing changes in health technology, the registrars should also have ongoing training sessions to relay updates. In 
conjunction, registrars need feedback. In the case of VCUHS, registrars were creating errors, HIM would fix them and no one was relaying that information back to them. Registrars would continuously repeat the same mistakes since they were unaware. Feedback is now provided and although the numbers of duplicate generated did not show a decline, the number of repeat registrars did. Lastly, and possibly the most important item, is that the MPI needs proper and routine analysis to query for errors. Addressing the need for ongoing cycle of analysis of errors and feedback and training will continue to be the focus in the future. Although a current workaround was implemented, this is a huge barrier to see real improvements. The barrier that exists is a lack of a current reporting option that is user friendly and not full of false positives. Consequently, VCUHS is abnormally complicated in essentially every aspect of the patient registration and MPI correction processes unlike other comparable institutions.

Overall, the underlying goals of a data integrity program should be more than a onetime fix and should involve an ongoing cycle of assessing, education, monitoring, and feedback. And finally, a truly successful program demands strong commitment and support from all stakeholders including executive leadership. Data integrity is everyone's job and as Bassett (2011) indicates that while the cause of these errors is usually easily explained, the solution is often anything but. 


\section{References}

Altendorf, Robin L. "Establishment of a Quality Program for the Master Patient Index." AHIMA's 79th National Convention and Exhibit Proceedings, October 2007.

Basset, Mike. 2011. "How to Measure Duplicate Rates. For The Record". Vol. 25 No. 7 P. 18

Bresnick, Jennifer. 2013. Healthcare Analytics Essentials: The master patient index. HealthITAnalytics. Accessed from: http://healthitanalytics.com/news/healthcareanalytics- essentials- the-master -patient-index.

Dimick, Chris. "Exposing Double Identity at Patient Registration." Journal of AHIMA 80, no.11 (November 2009)

Just, Beth. 2009. "Managing the Integrity of Patient Identity in Health Information Exchange " Journal of AHIMA 80, no.7: 62-69.

Just Associates, Inc. 2011. Duplicate Records Compromise EHR Investment.

Kuehn, Lynn. 1997. Health Information Management: Medical Records Process in Group Practice. Englewood, California. Center for Research in Amb. Health Care Admin.

Morris, Genevieve \& Greg Farnum. 2014. Patient Identification and Matching Final Report. Office of the National Coordinator for Health Information Technology. Accessed from: https://www.healthit.gov/sites/default/files/patient identification matching final rep ort.pdf

Purkis, Ben; Morris, Genevieve. 2012. Master Data Management within HIE Infrastructures: A Focus on Master Patient Indexing Approaches. Office of the National Coordinator for Health Information Technology. Accessed from: https://www.healthit.gov/sites/default/files/master data management final.pdf

VCU Health. (2015) About Us. Accessed from: http://www.vcuhealth.org/?id=5\&sid=1

Wheatley, V. 2008. "Quality Impact of the master patient index". Journal of AHIMA 79, no.10: 78-79. 
Williams, Kelly; Robinson, Kerin; Toth, Alexandra. 2006. Data quality maintenance of the patient master index: a snapshot of public healthcare facility PMI data quality and linkage activities. Journal of Health Info Management. Vol. 35 No. 1.

World Health Organization. 2007. "Patient Identification". Patient Safety Solutions vol 1. no. 2 
Appendix A: Illustration of VCUHS interface map.

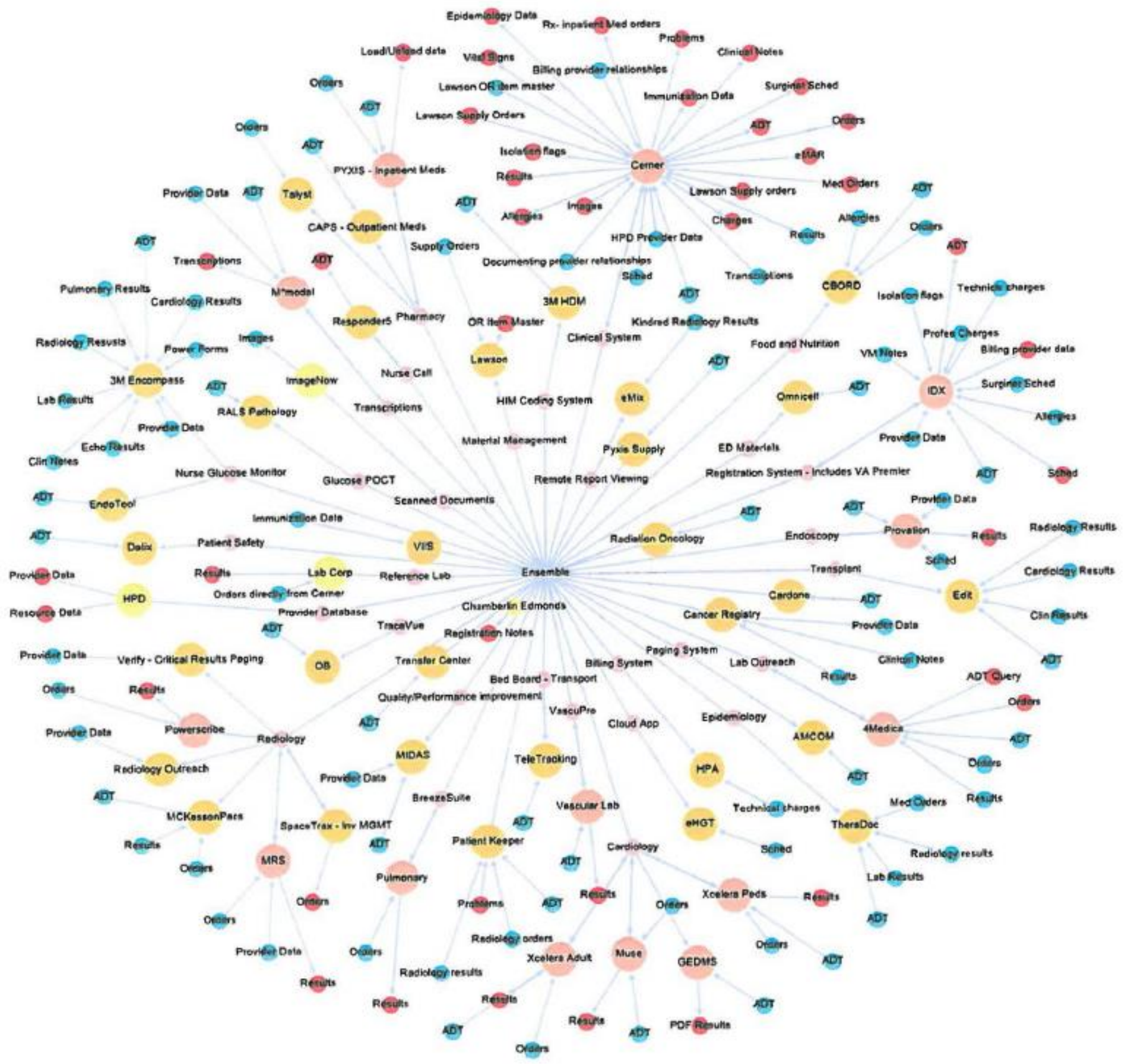

